\title{
SUBMANIFOLDS OF EUCLIDEAN SPACE WITH PARALLEL MEAN CURVATURE VECTOR
}

\author{
TAHSIN GHAZAL and SHARIEF DESHMUKH \\ Department of Mathematics \\ College of Science \\ P.O. Box 2455 \\ King Saud University \\ Riyadh 11451, Saudi Arabia \\ (Received November 21, 1989 and in revised form October 19, 1990)
}

ABSTRACT. The object of the paper is to study some compact submaniforlds in the Euclidean space $R^{n}$ whose mean curvature vector is parallel in the normal bundle. First we prove that there does not exist an n-dimensional compact simply connected totally real submanifold in $\mathrm{R}^{2 \mathrm{n}}$ whose mean curvature vector is parallel. Then we show that the $n$-dimensional compact totally real submanifolds of constant curvature and parallel mean curvature in $\mathrm{R}^{2 \mathrm{n}}$ are flat. Finally we show that compact Positively curved submanifolds in $R^{n}$ with parallel mean curvature vector are homology spheres. The last result in particular for even dimensional submanifolds implies that their Euler poincare' characteristic class is positive, which for the class of compact positively curved submanifolds admiting isometric immersion with parallel mean curvature vecto: in $R^{n}$, answers the problem of Chern and Hopf.

KEY WORDS AND PHRASES. Submanifolds, totally real submanifolds, homology sphere, Euler-poincare' characteristic. 1980 AMS SUBJECT CLASSIFICATION: 53C21, 53C25, 53C40.

1. Let $g$ be the flat metric of $R^{n}, \bar{\nabla}$ be the corresponding Riemannian connection. If $M$ is a submanifold of $R^{n}$ with normal bundle $v$, then the connection $\bar{\nabla}$ induces the Riemannian connection 
$\nabla$ on $M$ and the connection $\nabla^{\perp}$ in the normal bundle $\nu$, and we have $\bar{\nabla}_{X} Y=\nabla_{X} Y+h(X, Y), \bar{\nabla}_{X}{ }^{N=-A_{N}} X+\nabla_{X} N, X, Y \in X(M), N \varepsilon v$

where the second fundmental form $h(X, Y)$ is related to $A_{N} X$, by $g(h(X, Y), N)=g\left(A_{N} X, Y\right)$ and $X(M)$ is the Lie-algebra of vector fields on $M$. The mean curvature vector $H$ of $M$ is given by $H=$ $1 / n \quad \sum_{i=1}^{n} h\left(e_{i}, e_{i}\right)$, where $\left\{e_{1}, e_{2}, \ldots, e_{n}\right\}$ is a local orthonormal frame of $M$. The mean curvature vector $H$ is said to be parallel if $\nabla_{X} H=0, X \in X(M)$. If $H=0$ at each point of $M$, then $M$ is said to be a minimal submanifold. It is known that if $M$ is a compact submanifold of $R^{n}$, then $M$ is not a minmal submanifold (cf.[1]).

The even dimensional Euclidean space $R^{2 n}$ has complex structure $J$ which is parallel with respect to the connection $\bar{\nabla}$ that is, $R^{2 n}$ is a kaehler manifold. A submanifold $M$ of $R^{2 n}$ is said to be totally real if JTM $\subseteq \nu$, where TM is the tangent bundle of $M$. In the case $\operatorname{dim} M=n$ and $M$ is totally real submanifold of $R^{2 n}$, using (1.1), we obtain

$$
\frac{1}{\nabla_{X} J Y}=J \nabla_{X} Y \text { and } h(X, Y)=J A_{J Y} X, X, Y \in X(M) \text {. }
$$

2. In this section we study the n-dimensional totally real submanifold $M$ of $R^{2 n}$ with parallel mean curvature vector $H$, first under the topological restriction on $M$ that, it is compact and its first Betti number is zero, and then under the geometric restriction that it is a space of constant curvature.

THEOREM 2.1. There does not exist an n-dimensional compact totally real submanifold with first Betti number equal to zero and with parallel mean curvature vector in $\mathrm{R}^{2 \mathrm{n}}$.

PROOF. Let $M$ be an $n$-dimensional compact totally real submanifold of $\mathrm{R}^{2 \mathrm{n}}$ with parallel mean curvature vector $\mathrm{H}$. Then $\mathrm{JH}$ is a parallel vector field on $\mathrm{M}$.

The 1 -form $n$ dual to $\mathrm{JH}$ is also parallel and hence harmonic. If $\mathrm{H}^{1}(\mathrm{M} ; \mathrm{R})=0$, then $\eta$ and hence $\mathrm{H}$ must vanish. But this would mean that $M$ is a compact minimal submanifold of $R^{2 n}$, which is impossible (cf. [1]).

THEOREM 2.2. Let $M$ be an n-dimensional $\left(\begin{array}{lll}n & 2 & 2\end{array}\right)$ compact totally real submanifold of constant curvature in $R^{2 n}$ with parallel mean curvature vector. Then $M$ is flat. 
PROOF. If the curvature is nonzero constant, then $M$ is irreducible and cannot admit a nonzero parallel vector field JH.

3. In this section we shall be concerned with the positively curved submanifolds with parallel mean curvature vector in $R^{n}$. We prove the following.

THEOREM 3.1. Let $M$ be a compact and connected positively curved submanifold with parallel mean curvature vector in $R^{n}$. Then $M$ is a homology sphere.

PROOF. Sicne $M$ is compact, connected and $\nabla_{X^{H}}=0$, the function $\alpha=\mathbb{H} \mathbf{I}$ is a non-zero constant. Define the unit normal vector field $N$ on $M$ by $N=1 / \alpha$ H. If $\Phi: M \rightarrow R^{n}$ is the immersion of $M$ as submanifold of $R^{n}$, then the height function $f_{N}: M \rightarrow R$ is defined by $f_{N}(p)=g(\phi(p), N)$. The hessian of the height function at a critical point $p \in M$ of $f_{N}$ is given by the weingarten map $A_{N}$ at $p$. The curvature tensor $R$ of $M$ is given by $R(X, Y ; Z, W)=g(h(Y, Z), h(X, W))-g(h(X, Z), h(Y, W))$, from which the Ricci tensor Ric of $M$ is obtained as

$$
\operatorname{Ric}(X, Y)=n g(h(X, Y), H)-\sum_{i=1}^{n} g\left(h\left(X, e_{i}\right), h\left(Y, e_{i}\right)\right),
$$

where $\left\{e_{1}, e_{2}, \ldots, e_{n}\right\}$ is a local orthonormal frame of $M$. Sicne $M$ is positively curved and for a unit vector field $X, \operatorname{Ric}(X, X)$ is the sum of the sectional curvatures, $\operatorname{Ric}(X, X)>0$. Thus from (3.1) we obtain $g(h(x, X), H)>0$. This given that $g\left(A_{N} X, X\right)>0$, for each unit vector field $x \in \times(M)$. This proves that all the eigenvalues of $A_{N}$ are positive at each point of $\dot{M}$. Thus the height function $f_{N}$ has no non-degenerate critical points of index $i=1,2, \ldots, n-1$. Using Morse inequalities we obtain

$$
H^{1}(M, R)=0, \ldots \ldots, H^{n-1}(M, R)=0 .
$$

Since $M$ is compact, we get that $M$ is a homology sphere.

COROLLARY 3.1. The real projective space $\mathrm{RP}^{\mathrm{m}}$ and the complex projective space $\mathrm{CP}^{2}$ cannot be isometrically immersed in $\mathrm{R}^{\mathrm{n}}$ with parallel mean curvature vector.

Combining Theorem 2.1 with Theorem 3.1 , we get

COROLLARY 3.2. There does not exist an n-dimenstional compact and connected positively curved totally real submanifold in $\mathrm{R}^{2 \mathrm{n}}$ with parallel mean curvature vector. 
Remark. The Chern-Hopf problem is "The Euler-poincare" characteristic class of an even dimensional positively curved manifold $M$ satisfies $x(M)>0 "$. For class of even dimensional positively curved compact and connected manifolds which admit isometric commersion in $\mathrm{R}^{\mathrm{n}}$ with parallel mean curvature vector we have the following corollary to Theorem 3.1 .

COROLLARY 3.3. Let $M$ be an even dimensional compact and connected positively curved submanifold of $R^{n}$ with parallel mean curvature vector. Then $x(M)>0$.

\section{ACKNOWLEDGEMENT}

The research is supported by the grant No. (Math/1409/05) of the Research Center, College of Science, King Saud University

\section{REFERENCES}

1. Kobayashi, S. and Nomizu, K., Foundations of differential geometry, Vol II, Interscience tract, New York, (1069).

2. Milnor, J., Morse Theory, Ann. of Math. Studies, princeton University press, Princeton, (1963).

3. Weinstein, A., Positively Curved $n$-manifolds in $R^{n+2}$, J. Diff Geom. 4, 1-4 (1970). 


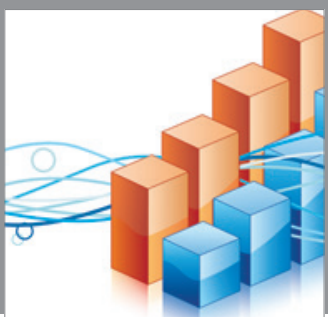

Advances in

Operations Research

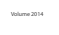

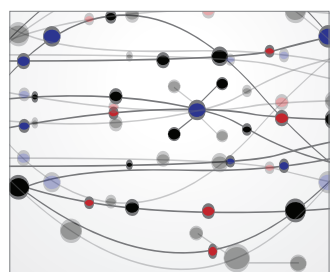

\section{The Scientific} World Journal
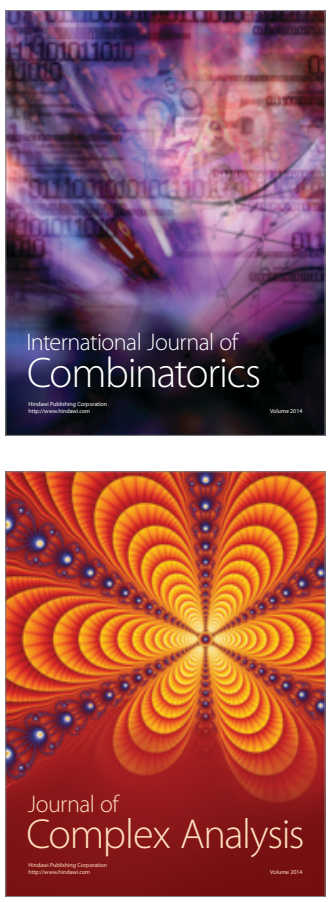

International Journal of

Mathematics and

Mathematical

Sciences
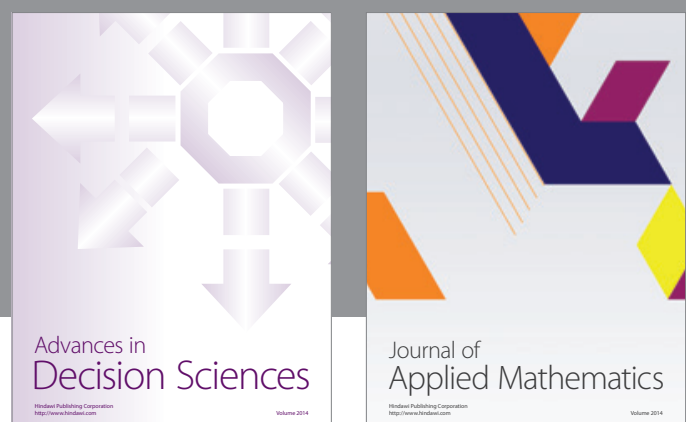

Journal of

Applied Mathematics
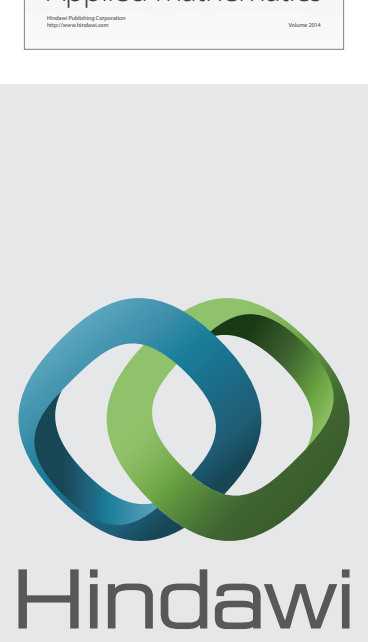

Submit your manuscripts at http://www.hindawi.com
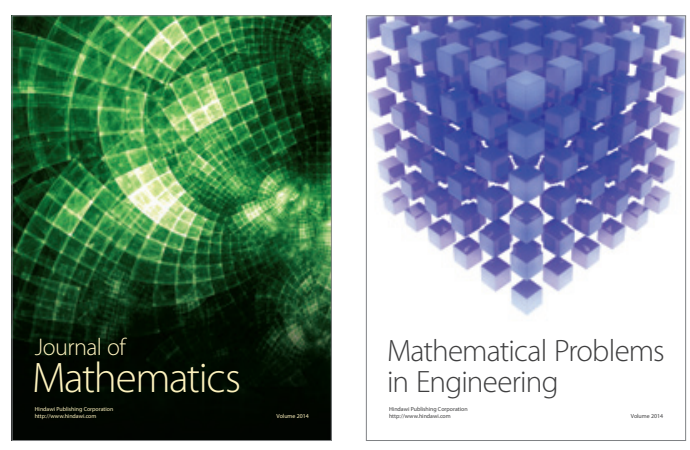

Mathematical Problems in Engineering
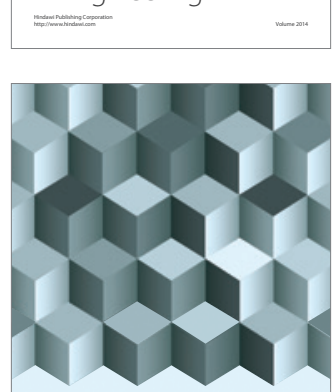

Journal of

Function Spaces
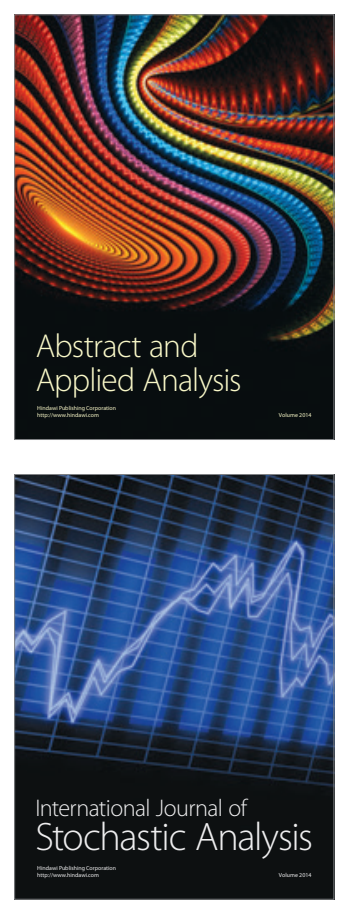

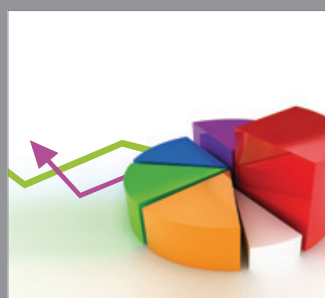

ournal of

Probability and Statistics

Promensencen
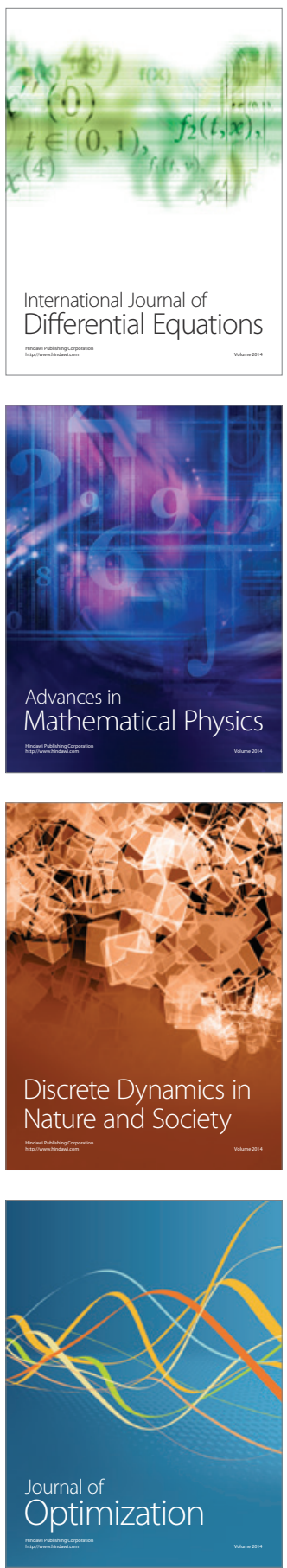